\title{
Karl Mannheim und das Problem der historischen Zeit
}

\section{Klaus Lichtblau}

\section{Karl Mannheim und das Problem der historischen Zeit}

Karl Mannheim hat wiederholt darauf hingewiesen, daß sich zentrale Motive seiner Wissenssoziologie der Erfahrung des Historismus verdanken. Für ihn war es deshalb selbstverständlich, daß auch die verschiedenen Varianten der modernen Soziologie von den sozialen und weltanschaulichen Strömungen ihrer Zeit geprägt waren. Der von Mannheim als unvermeidbar angesehene Kampf der modernen Weltanschauungen um die Meinungsführerschaft war insofern nicht nur Gegenstand seiner wissenssoziologischen Arbeiten, sondern zugleich eine zentrale Herausforderung für sein eigenes Projekt einer wissenssoziologischen Zeitdiagnose. Wie alle modernen Weltanschauungen hatte sich auch seine Wissenssoziologie gegenüber konkurrierenden Weltdeutungen zu bewähren. Mannheim zufolge kann dabei diejenige Theorie als die adäquateste betracht werden, die den umfassendsten Blick auf eine gegebene historische Konstellation ermöglicht. Auch für Mannheims Wissenssoziologie trifft deshalb das Kriterium der „Seinsverbundenheit“ zu. Als „seinsverbunden“ bezeichnete er ein Denken, bei dem das Ergebnis vom jeweiligen Standpunkt des Beobachters abhängig ist. Seinsverbundenheit schließt zwar die Möglichkeit einer diskursiven Begründung von Erkenntnissen nicht aus. Sie macht jedoch deutlich, daß logische Geltung und soziale Akzeptanz eines Denkens nicht identisch sind. Beobachterstandpunkte können zum einen je nach sozialer Lage differieren. So denken Angehörige von privilegierten Schichten Mannheim zufolge in der Regel anders als Angehörige von Unterschichten. Beobachterstandpunkte können aber auch gemäß dem für sie jeweils typischen inneren Zeiterleben voneinander abweichen. Mannheim machte diesen Tatbestand sowohl am Phänomen des Zusammenlebens verschiedener Generationen als auch am unterschiedlichen Zeiterleben des „konservativen“ und des „progressiven“ Denkens deutlich. Koexistierende Generationen mögen zwar in derselben chronometrischen Zeit leben. Dies schließt jedoch nicht aus, daß sie diese gemäß völlig unterschiedlichen inneren Erlebniszeiten wahrnehmen und interpretieren. In gleicher Weise differiert auch das Zeiterleben des „konservativen“ und des „progressiven“ Menschen: Für ersteren ist die Gegenwart der Endpunkt einer glorreichen Vergangenheit, für den letzteren dagegen der Ausgangspunkt für zukünftige Höherentwicklungen der Menschheit. Sowohl für die Generationslagerung als auch für das unterschiedliche Gegenwartserleben des Konservativen und des Progressiven ist also eine „Ungleichzeitigkeit des Gleichzeitigen“ kennzeichnend, die auf zentrale Unterschiede der damit jeweils einhergehenden historischen Zeiterfahrungen verweist.[1]

Der von Mannheim in den Mittelpunkt seiner Analysen gestellte Perspektivismus der jeweiligen sozialen Lage ist insofern auch durch ein unterschiedliches Zeitempfinden der einzelnen sozialen Gruppen und Generationen geprägt. Ist es vor diesem Hintergrund modernen Gesellschaften überhaupt möglich, ein einheitliches Zeitbewußtsein zu entwickeln? Und welchen Beitrag leistete die Mannheimsche Wissenssoziologie zur Klärung der epochalen Selbstvergewisserung seiner Zeit? Denn daß die moderne Denkkrisis zugleich untrennbar mit einer Krise des historischen Weltbildes verbunden ist, an dessen Ausarbeitung verschiedene Generationen seit Jahrhunderten beteiligt gewesen sind, war eine Überzeugung, die Karl Mannheim mit Ernst Troeltsch teilte und die ihn 
überhaupt erst zu seinen wissenssoziologischen Forschungen motiviert hat.[2] Im Unterschied zu Troeltsch hatte Mannheim jedoch nicht mehr die Hoffnung, daß es jemals möglich sei, die geschichtliche Bedingtheit unseres Denkens durch den weiteren Verlauf der Geschichte selbst zu überwinden. Die moderne wissenssoziologische Forschung ist ihm zufolge allenfalls in der Lage, die Genese und den Strukturwandel unseres geschichtlichen Weltbildes bis hin zur gegenwärtigen Konstellation verständlich zu machen und die dabei anstehenden epochalen Entscheidungen zu verdeutlichen.

Mannheim machte in diesem Zusammenhang den Vorschlag, die im Laufe der europäischen Neuzeit entwickelten unterschiedlichen Auffassungen der historischen Zeit durch den Gestaltwandel des utopischen Bewußtseins zu erklären. Dies ist weder selbstverständlich noch trivial, da Mannheim mit diesem Vorschlag die Geschichte des neuzeitlichen Denkens in einen größeren religionsgeschichtlichen Zusammenhang gestellt hat. Es ist insofern kein Zufall, daß Mannheim auch die großen weltanschaulichen Auseinandersetzungen seiner Epoche in einer kryptoreligiösen Sprache beschrieben hat. Utopien stellen jedoch in der Regel „Wunschräume“ dar, während Mannheim primär an einer Erklärung des Phänomens der Verzeitlichung des Bewußtseins interessiert war. Im Mittelpunkt seiner diesbezüglichen Untersuchung über das utopische Bewußtsein standen deshalb jene „menschlichen Sehnsuchtsprojektionen“, die sich in der Vorstellung bestimmter „Wunschzeiten“ entladen und die man auch als Chiliasmen zu bezeichnen pflegt. Mannheim betonte in diesem Zusammenhang ausdrücklich den chiliastischen Ursprung des neuzeitlichen Bewußtseins, dessen geschichtliche Entwicklung er in Gestalt eines zunehmenden Spannungsverlustes zwischen der jeweils vorherrschenden „seinstranszendenten Vorstellung“ und der gegebenen Wirklichkeit beschrieb.[3] Als historisch gelten dieser Überlegung zufolge dabei nur jene Zeitvorstellungen, bei denen die Wunschzeit mit der gegenwärtigen Zeit nicht identisch ist, die utopischen Energien aber nicht mehr auf das Jenseits gerichtet sind, sondern selbst die Wirklichkeit umzugestalten beginnen. Oder anders gesprochen: „Wie eine konkrete Gruppe, wie eine soziale Schicht die historische Zeit gliedert, das hängt von ihrer Utopie ab.“[4] Utopien sind also zentrale Bestandteile der jeweiligen Geschichtsphilosophie, vermittels der überhaupt erst eine qualitative Differenzierung der historischen Zeit möglich wird. Aus der empirischen Geschichtsbetrachtung kann letztere nicht gewonnen werden; vielmehr stellt sie deren oft unhinterfragte Voraussetzung dar.5]

Mannheim sah die Wende von einer strikten Jenseitsorientierung hin zu einer innerweltlichen Erlösungshoffnung bereits in den millenaristischen Bewegungen der Reformationszeit vollzogen, die ohne weitere zeitliche Verzögerung bereits in der Gegenwart das Gottesreich auf Erden zu verwirklichen versucht hatten. Diese Vorstellung eines unmittelbar bevorstehenden Einbruchs des Ewigen in die Gegenwart war jedoch noch völlig unhistorisch. Denn die einzige zeitliche Unterscheidung, die sie gelten ließ, war die zwischen einer „sinnfremden“ und einer „sinnerfüllten Zeit“. Der Millenarismus der täuferischen Bewegung stellt Mannheim zufolge dabei die radikalste Form dar, in der seinstranszendente Vorstellungen die Wirklichkeit umzugestalten vermögen. Obgleich sich dieser Radikalismus noch genuin religiösen Motiven verdankte, interpretierte Mannheim das Täufertum und die Bauerkriege der Reformationszeit als die erste neuzeitliche Erscheinungsform der modernen sozialen Bewegungen. Gleichwohl stellt der täuferische Millenarismus Mannheim zufolge in jeder Hinsicht einen historischen Grenzfall dar, weil sich seine utopischen Vorstellungen ekstatischen Visionen verdanken, die bewußt von jedem Raum- und Zeitbezug abstrahieren, weshalb Mannheim die Nähe dieser Visionen zur mystischen Erfahrung hervorgehoben hat.[6]

Ausgangspunkt von Mannheims Rekonstruktion der Entwicklungsdynamik des neuzeitlichen 
Denkens ist also eine völlig ahistorische Vision einer „sinnerfüllten Zeit“, die sich radikal von der Gegenwart unterscheidet und die jederzeit eintreten kann. Ihre Realisierung ist dabei an keine geschichtlichen Voraussetzungen gebunden, sondern kann hier und jetzt stattfinden. Die geschichtsphilosophische Ausgestaltung dieser chiliastischen Sehnsüchte der Menschheit hatte in der Folgezeit zu einer Transformation des utopischen Bewußtseins geführt, die zugleich für den zunehmenden Spannungsverlust zwischen Utopie und Wirklichkeit verantwortlich ist. Mannheim erklärte die damit einhergehende Verzeitlichung des neuzeitlichen Denkens durch die Art und Weise, wie in den einzelnen Weltanschauungen das Verhältnis zwischen der Gegenwart und der kommenden sinnerfüllten Zeit jeweils konkret interpretiert wurde.

Die großen weltanschaulichen Strömungen, die im Laufe der europäischen Neuzeit zentrale Beiträge für die Ausgestaltung unseres historischen Weltbildes geleistet haben und mit denen sich Mannheim ausführlicher beschäftigt hat, sind der Liberalismus, der Konservatismus und der Sozialismus. Innerhalb der Geschichte des utopischen Bewußtseins stellt der Konservatismus jedoch einen Grenzfall dar, auf den gleich noch einzugehen sein wird. Am anderen Ende dieser neuzeitlichen Bewußtseinsgeschichte stehen Mannheim zufolge dagegen der sozialrevolutionäre Anarchismus, der Faschismus und das „amerikanische Bewußtsein“, die aufgrund ihrer eigenen unhistorischen Betrachtungsweise in jeweils unterschiedlicher Weise dieses im Laufe der Neuzeit entstandene historische Weltbild grundsätzlich in Frage gestellt haben. Mannheim sah diese Lage als so bedrohlich an, daß er Ende der zwanziger Jahre diesbezüglich sogar eine schicksalhafte Entscheidungssituation gegeben sah, von deren Ausgang die weitere Entwicklung unseres historischen Bewußtseins abhängig sei.

Kennzeichnend für den Liberalismus ist die Vorstellung, daß die Geschichte einen kontinuierlichen Fortschrittsprozeß darstellt. Anstelle der Jenseitsorientierung ist bei ihm die optimistische Erwartung getreten, daß sich die liberale Utopie zwar nicht sofort in der Wirklichkeit realisieren läßt, daß sich aber die Wirklichkeit bereits auf dem Weg hin zur Realisierung dieses Ziels befindet. Die historische Zeit wird dabei als linearer Fortschritts- und Entwicklungsprozeß gedeutet, der im Prinzip berechenbar geworden ist und deshalb keine unliebsamen Überraschungen mehr beinhaltet. Die liberale Utopie ist insofern mit der Unendlichkeit des „Fortschritts“ identisch, zu dem es keine vernünftige Alternative gibt.[7]

Auch die von Karl Marx und Friedrich Engels begründete sozialistische Weltanschauung teilt noch viele Eigenschaften mit diesem liberalen Geschichtsbild. Auch hier wird der geschichtliche Verlauf als ein Fortschrittsprozeß gedeutet, bei dem sich Phasen einer kontinuierlichen gesellschaftlichen Entwicklung und Phasen der Krise sowie der sozialen Erschütterung regelmäßig abwechseln. Anspruch von Marx und Engels war es, den Übergang vom Kapitalismus zum Sozialismus als notwendig erwiesen und bis zu einem bestimmten Grad zugleich berechenbar gemacht zu haben. Zwar gibt es auch ihnen zufolge einen gewissen Spielraum für die revolutionäre Aktion, um den Übergang vom Kapitalismus zum Sozialismus zu beschleunigen. Allerdings ist in ihren Augen eine erfolgreiche politische Revolution an gesellschaftliche Rahmenbedingungen gebunden, über die man sich nicht beliebig hinwegsetzen kann. Die anarchistische Vorstellung, daß der Ausbruch der Revolution von keinen sozialen „Gesetzmäßigkeiten“ abhängig sei, sondern jederzeit stattfinden könne, lehnten sie deshalb entschieden ab. Mannheim deutete die marxistische Revolutionstheorie dergestalt, daß in ihr die Vorstellung der Revolte einen „bürokratischen Einschlag“ bekommen habe. Zwar hätten auch Marx und Engels sich nicht ganz von der Vorstellung befreien können, daß es einen „günstigen Augenblick“ für die erfolgreiche revolutionäre Tat gebe, der nicht eindeutig vorhersehbar sei und der insofern die grundsätzliche Irrationalität der politischen Sphäre unterstreiche. Allerdings sei es ihr Ziel gewesen, das Irrationale so weit wie möglich zu rationalisieren, um das Schicksal der 
Revolte nicht ganz von einer Augenblickssituation abhängig zu machen. Mannheim charakterisierte deshalb die marxistische Geschichtsphilosophie als „das rationale Denken der irrationalen Tat“, wobei das Geschichtserleben zu einem „wahren strategischen Plan“ geworden sei.[8] Während sich die utopischen Vorstellungen des Liberalismus und Sozialismus prinzipiell an der Zukunft orientieren, beruht die konservative Utopie dagegen auf einer Neubewertung der Vergangenheit. Ihr zufolge sind Vergangenheit und Gegenwart gar nicht radikal voneinander geschieden, sondern die Vergangenheit reicht in die Gegenwart hinein und bestimmt auch noch das aktuelle Erleben und Handeln. Der revolutionäre Bruch mit der Tradition wird bewußt abgelehnt, und auch die Entwertung der Gegenwart zugunsten von geschichtsphilosophisch begründeten utopischen Zukunftsvorstellungen wird verworfen. Das konservative Denken besitzt deshalb im Grunde genommen weder eine Utopie noch eine Geschichtsphilosophie: seine „Utopie“ ist vielmehr eine reine Gegen-Utopie. Denn das Utopische ist ihm zufolge in der Welt selbst bereits präsent: „Sinnziel und Wirklichkeit, Sollen und Sein fallen hier nicht auseinander.“[9] Konservatives Denken verdankt sich deshalb nicht nur der Entdeckung der Bedeutung der Vergangenheit, sondern zugleich der „Entdeckung der Werte zeugenden Zeit“.[10]

Das konservative Zeiterleben beruht dabei auf einer Neubewertung des Dauerhaften in Gestalt eines „virtuellen Präsentsein der Vergangenheit“.[11] Denn es behandelt die Vergangenheit so, als ob sie noch nicht abgeschlossen sei. Mannheim sah darin eine methodische Paradoxie, da es auch dem konservativen Denken nicht gelinge, das vergangene Leben in gleicher Weise wie das aktuelle Erleben zu vergegenwärtigen. Er unterschied deshalb den Konservatismus begrifflich vom Traditionalismus, weil dieser im Unterschied zum Traditionalismus durch eine reflexive Denkhaltung gekennzeichnet ist. Denn reflexiv ist sowohl sein Verhältnis zum Liberalismus, von dem er sich polemisch abgrenzt, als auch sein Versuch einer Vergegenwärtigung vergangenen Lebens. In letzterer Hinsicht hat insbesondere die Romantik entscheidende Beiträge zur Entwicklung der historischen Geistes- und Kulturwissenschaften geleistet. Dies ist auch der Grund, weshalb Mannheim den Historismus zumindest in methodologischer Hinsicht als legitimen Erben der konservativen Denkströmung angesehen hat.[12] Konservatives Denken ist dieser Auffassung zufolge zwar historisch, aber nicht geschichtsphilosophisch ausgerichtet. Denn es verzichtet auf eine Abwertung der Vergangenheit zugunsten der Gegenwart ebenso wie auf eine Abwertung der Gegenwart zugunsten der Zukunft. Dies besagt ja das bekannte Diktum Leopold von Rankes, daß alle Epochen in demselben unmittelbaren Verhältnis zu Gott stünden und insofern nicht gegeneinander ausgespielt werden dürften.

Zwar hat der durch die konservative Denkströmung geprägte Historismus in unvergleichbarer Weise unser historisches Wissen bereichert. Dennoch stellt er eine radikale Infragestellung des geschichtsphilosophischen Denkens dar, weil er auf eine entsprechende Bewertung der einzelnen Epochen bewußt verzichtet. Obgleich die Mannheimsche Wissenssoziologie selbst in der Tradition des Historismus steht, hat sie sich aus diesem Grund dessen relativistischen Konsequenzen nicht zu eigen gemacht. Statt dessen hat sie den Versuch übernommen, die der dialektischen Geschichtsbetrachtung Hegels zugrunde liegende Vorstellung einer werdenden Totalität in abgeschwächter Form für eine wissenssoziologische Zeitdiagnose fruchtbar zu machen. Die dialektische Methode Hegels stellt Mannheim zufolge eine gelungene Synthese zwischen einem radikalen Historismus und einer zeitlosen begrifflichen Systematik dar. Die einzige Korrektur, die Mannheim an Hegels Dialektik vornahm, besteht darin, daß Mannheim es ausschloß, daß die Entwicklungsgeschichte des neuzeitlichen Bewußtseins eines Tages in einer „absoluten Synthese“ der einzelnen Denkströmungen ihren glorreichen Abschluß findet. Mannheim hielt vielmehr immer 
nur „relative Synthesen“ für möglich, in die das ganze Wissen einer Epoche einging und die dennoch keinen dauerhaften Bestand haben, weil ihm zufolge der geschichtliche Prozeß zu keinem definitiven Abschluß kommt.[13]

Auch die Mannheimsche Wissenssoziologie stellt insofern keine „absolute“ Form des Wissens dar. Das einzige, was sie für sich in Anspruch nahm, war, daß sie auf der Höhe ihrer Zeit stand und in einem größtmöglichen Umfang den gegensätzlichen Strömungen innerhalb der modernen Weltanschauungen Rechnung trug. Dies setzt die Einlösung eines gewissen Vollständigkeitsanspruchs voraus, der allerdings nicht absolut, sondern historisch-konkret gemeint war. Ausgehend von einer empirischen Bestandsaufnahme der einzelnen Denkströmungen war es Mannheim zufolge durchaus möglich, die Krise des modernen Denkens zu überwinden, um so die Grundlage für ein neues Entfaltungsstadium des menschlichen Geistes zu schaffen. Dies war allerdings nur dann der Fall, wenn innerhalb einer solchen Bestandsaufnahme auch diejenigen Positionen zur Sprache kamen, die den Sinn einer historischen Weltbetrachtung grundsätzlich in Frage stellten.

Zu diesen radikalen Gegenströmungen zählte Mannheim auch den französischen Syndikalismus und den italienischen Faschismus, die ihm zufolge in einem engen geistesgeschichtlichen Zusammenhang stehen. Kennzeichnend für sie sei der Glaube, daß der mögliche Erfolg einer politischen Revolte an keine geschichtsphilosophischen Voraussetzungen gebunden sei, sondern sich günstigen Umständen sowie der Entschlossenheit zur Tat verdanken. Der ganze geschichtliche Prozeß verschmelze hier zu einem „Augenblick“, den es zu erkennen gilt und dessen Eintreten sich keiner erkennbaren historischen Logik, sondern dem reinen Zufall verdankt. Es handele sich hierbei um die Ideologie putschistischer Gruppierungen, für die alle geschichtsphilosophischen Konstruktionen reine „Mythen“ seien, deren Sinn allein darin bestehe, für den politischen Kampf funktionalisiert zu werden. Der revolutionäre Syndikalist und der Faschist glaube deshalb auch nicht an den linearen Charakter des historischen Prozesses, sondern orientiere sich an der Vorstellung, daß der Aufstieg und der Niedergang einer Kultur vielmehr einem zyklischen Muster folgt, das es zu erkennen gilt, weil es in der Geschichte ständig wiederkehrt. An die Stelle der Geschichtsphilosophie sei im faschistischen Denken deshalb nicht zufällig Nietzsches Lehre von der ewigen Wiederkehr des Gleichen und Paretos Theorie der Elitenzirkulation getreten. Auch wenn der Faschismus gegenüber dem im Laufe der europäischen Neuzeit entwickelten historischen Weltbild Mannheim zufolge eine „Reprimitivisierung“ darstellt, nahm er diese Bestrebungen sehr ernst, weil sie ihrerseits einen Ausweg aus der Krise der Moderne aufzeigten, auch wenn dieser direkt in die Diktatur führte.[14] Die zweite große Gefahr, die dem historischen Weltbild drohte, dem sich Mannheims Wissenssoziologie verpflichtet fühlte, wurde von ihm als „amerikanisches Bewußtsein“ bezeichnet. Mannheim spielte mit diesem Begriff zum einen auf die Besonderheit der Besiedelung des nordamerikanischen Kontinents an, die Jahrhunderte lang durch keine natürlichen Grenzen eingeschränkt worden ist und insofern die Ausbildung eines entsprechenden Bewußtseins begünstigt hat, daß alle Probleme mit organisatorischen und technischen Mitteln lösbar seien. Zum anderen meinte Mannheim damit eine Bewußtseinshaltung, die sich zu seiner Zeit auch innerhalb der Soziologie zunehmend durchzusetzen begann. Er hatte dabei die Tendenz im Auge, historische Sinnzusammenhänge auf „ewiggleiche Gesetzmäßigkeiten“ und auf die Kombination von „ein für allemal feststellbare Typenstrukturen“ zu reduzieren, wie sie für bestimmte Spielarten der modernen Soziologie charakteristisch sei.[15]

Typisch für Mannheim ist es, daß er nicht nur das Bedrohliche solcher Entwicklungen wahrnahm, sondern auch die neuen Möglichkeiten sah, die sie eröffneten. Er konnte es sich nämlich durchaus vorstellen, daß eine fruchtbare Synthese zwischen seinen eigenen wissenssoziologischen 
Forschungen und der modernen amerikanischen Soziologie möglich sei, sollte man ihm jemals die Chance geben, im nordamerikanischen Universitätssystem Fuß zu fassen.[16] Was ihn aber vor der Zeit der nationalsozialistischen Machtergreifung am meisten bewegte, war die Angst, daß jene besondere Konstellation, die zum ersten Mal einen tieferen Einblick in die Entwicklungsdynamik des neuzeitlichen Denkens gestattete, bald vorbei sein könnte und daß es deshalb darum ging, die Gunst des Augenblicks für einen ernsthaften Dialog zwischen den einzelnen weltanschaulichen Strömungen zu nutzen. Mit seinen eigenen wissenssoziologischen Arbeiten machte er dabei deutlich, daß die historischen Voraussetzungen hierfür gegeben waren. Und auch seine Diagnose hat sich bestätigt, daß innerhalb der Krisis des modernen Denkens die Zeit für eine Entscheidung längst reif war.

in: Bálint Balla, Vera Sparschuh \& Anton Sterbling (Hg.): Karl Mannheim. Leben, Werk, Wirkung und Bedeutung für die Osteuropaforschung. (Beiträge zur Osteuropaforschung, Bd. 13.) Hamburg: Reinhold Krämer Verlag 2007, S. 11-19

[1] Vgl. Karl Mannheim, Wissenssoziologie, 2. Aufl. Neuwied / Berlin 1970, S. 517.

[2] Vgl. Ernst Troeltsch, Der Historismus und seine Probleme, Tübingen 1922. Zu Mannheims Verhältnis zu Troeltsch vgl. ders., Wissenssoziologie, S. $246 \mathrm{ff}$.

[3] Karl Mannheim, Ideologie und Utopie, 5. Aufl. Frankfurt am Main 1969, S. 179 ff.

[4] Ebd., S. 182.

[5] Ebd., S. 196.

[6] Ebd., S. $184 \mathrm{ff}$.

[7] Ebd., $191 \mathrm{ff}$.

[8] Ebd., S. 116 und 213.

[9] Ebd. S., 201.

[10] Ebd., S. 203.

[11] Ebd., S. 204.

[12] Wissenssoziologie, S. 408 ff.; ders., Konservatismus. Ein Beitrag zur Soziologie des Wissens, Frankfurt am Main 1984.

[13] Ideologie und Utopie, S. 132; vgl. ferner Karl Mannheim, Strukturen des Denkens, Frankfurt am Main 1980, S. 44.

[14] Ideologie und Utopie, S. 116 ff.; vgl. ferner Mannheims Frankfurter Vorlesung vom SS 1930.

[15] Ideologie und Utopie, S. $218 \mathrm{f}$.

[16] Vgl. hierzu Mannheims Brief an Louis Wirth vom 26. Juli 1933, in: Mannheim Károly levelezése 19111946, Budapest 1996, S. 65 f.

C 2001-2003 Fachbereich Gesellschaftswissenschaften, Johann Wolfgang Goethe-Universität Frankfurt/Main 